\title{
THE COEFFICIENT COALGEBRA OF A SYMMETRIZED TENSOR SPACE
}

\author{
RANDALL R. HOLMES DAVID P. TURNER
}

\begin{abstract}
The coefficient coalgebra of $r$-fold tensor space and its dual, the Schur algebra, are generalized in such a way that the role of the symmetric group $\Sigma_{r}$ is played by an arbitrary subgroup of $\Sigma_{r}$. The dimension of the coefficient coalgebra of a symmetrized tensor space is computed and the dual of this coalgebra is shown to be isomorphic to the analog of the Schur algebra.
\end{abstract}

\section{INTRODUCTION}

Let $K$ be the field of complex numbers. The vector space $E=K^{n}$ is naturally viewed as a (left) module for the group algebra $K \Gamma$ of the general linear group $\Gamma=\mathrm{GL}_{n}(K)$. The $r$-fold tensor product $E^{\otimes r}$ is in turn a module for $K \Gamma^{r}$, where $\Gamma^{r}=\Gamma \times \cdots \times \Gamma$ ( $r$ factors). Let $G$ be a subgroup of the symmetric group $\Sigma_{r}$ and let $\chi: G \rightarrow K$ be an irreducible character of $G$. The "symmetrized tensor space" associated with $\chi$ is $E^{\chi}=E^{\otimes r} t_{\chi}$, where $t_{\chi}$ is the central idempotent of the group algebra $K G$ corresponding to $\chi$ (with the action of $G$ on $E^{\otimes r}$ being given by place permutation).

In this paper, we study the coefficient coalgebra $A_{\chi}$ of the $R$-module $E^{\chi}$, where $R$ is the subalgebra of $K \Gamma^{r}$ consisting of those elements fixed by $G$ under the action of $\Sigma_{r}$ on $\Gamma^{r}$ given by entry permutation. (If $V$ is an $R$-module with finite $K$-basis $\left\{v_{j} \mid j \in J\right\}$, then for each $\kappa \in R$, we have $\kappa v_{j}=\sum_{i} \alpha_{i j}(\kappa) v_{i}$ for some $\alpha_{i j}: R \rightarrow K$. The linear span of the functions $\alpha_{i j}$ $(i, j \in J)$ has a natural coalgebra structure. It is the "coefficient coalgebra" of $V$, denoted $\operatorname{cf}(V)$.)

Section 1 sets up notation and presents some standard results suitably generalized to the current situation.

In Section 2, we obtain generalizations of some classical results (i.e., results for the case $G=\Sigma_{r}$ ). In particular, we show that the image $S$ of the representation afforded by the $R$-module $E^{\otimes r}$ equals the set of those endomorphisms of $E^{\otimes r}$ that commute with the action of $G$ (Theorem 2.7). This generalizes Schur's Commutation Theorem [Ma, 2.1.3] in the classical case, where $S$ is the "Schur algebra." We also observe that the algebra isomorphism $S \cong A^{*}\left(A=\operatorname{cf}\left(E^{\otimes r}\right)\right)$ in the classical case [Ma, 2.3.5 and following paragraph] continues to hold with $G$ arbitrary (Theorem 2.8).

Date: April 26, 2011.

1991 Mathematics Subject Classification. 20G43, $16 \mathrm{~T} 15$. 
In Section 3, we study the coefficient coalgebra $A_{\chi}$ and the corresponding analog $S_{\chi}$ of the Schur algebra and establish an algebra isomorphism $S_{\chi} \cong$ $A_{\chi}^{*}$ (Theorem 3.4). We exhibit decompositions of $A$ and $S$ in terms of the various $A_{\chi}$ and $S_{\chi}$, respectively (Theorems 3.3 and 3.5), and end by providing a formula for the dimension of $A_{\chi}$ over $K$ (Theorem 3.6).

Some of the results of this paper appear in the Ph.D. dissertation [Tu] of the second author written under the direction of the first author. We thank the referee for some useful suggestions.

\section{Notation AND BACKGROUND}

Let $G$ be a fixed subgroup of the symmetric group $\Sigma_{r}$.

For the general results of this section and the next, the field $K$ can be more general than the field of complex numbers. We assume only that $K$ is an infinite field of characteristic not a divisor of $|G|$. Fix a positive integer $n$. The vector space $E=K^{n}$ is acted on naturally (from the left) by the semigroup $M=\operatorname{Mat}_{n}(K)$ of $n \times n$ matrices over $K$ and is therefore a $K M$ module, where $K M$ is the semigroup algebra of $M$ over $K$.

The group $G$ acts from the right on $M^{r}=M \times \cdots \times M$ ( $r$ factors) by $m \sigma=\left(m_{\sigma(1)}, m_{\sigma(2)}, \ldots, m_{\sigma(r)}\right)\left(m \in M^{r}, \sigma \in G\right)$.

The semigroup $M^{r}$ acts on $E^{\otimes r}=E \otimes \cdots \otimes E$ ( $r$ factors) by $m v=$ $m_{1} v_{1} \otimes \cdots \otimes m_{r} v_{r}\left(m \in M^{r}, v=v_{1} \otimes \cdots \otimes v_{r} \in E^{\otimes r}\right)$. This action extends linearly to make $E^{\otimes r}$ a $K M^{r}$-module.

For each $1 \leq \alpha \leq n$, let $e_{\alpha} \in E$ be the $n$-tuple with $\beta$-entry $\delta_{\alpha \beta}$ (Kronecker delta). Let $I=\left\{i=\left(i_{1}, \ldots, i_{r}\right) \in \mathbf{Z}^{r} \mid 1 \leq i_{j} \leq n\right.$ for all $\left.j\right\}$. The space $E^{\otimes r}$ has basis $\left\{e_{i} \mid i \in I\right\}$, where $e_{i}:=e_{i_{1}} \otimes \cdots \otimes e_{i_{r}}$.

For $m \in M^{r}$ and $i, j \in I$, define $m_{i, j}=\prod_{\alpha=1}^{r}\left(m_{\alpha}\right)_{i_{\alpha} j_{\alpha}}$.

1.1 Lemma. Let $m \in M^{r}$.

(i) $m e_{j}=\sum_{i} m_{i, j} e_{i} \quad(j \in I)$.

(ii) $(m \sigma)_{i, j}=m_{i \sigma^{-1}, j \sigma^{-1}} \quad(\sigma \in G, i, j \in I)$.

Proof. (i) For all $j \in I$, we have

$$
\begin{aligned}
m e_{j} & =m_{1} e_{j_{1}} \otimes \cdots \otimes m_{r} e_{j_{r}}=\left(\sum_{i_{1}=1}^{n}\left(m_{1}\right)_{i_{1} j_{1}} e_{i_{1}}\right) \otimes \cdots \otimes\left(\sum_{i_{r}=1}^{n}\left(m_{r}\right)_{i_{r} j_{r}} e_{i_{r}}\right) \\
& =\sum_{i \in I} \prod_{\alpha=1}^{r}\left(m_{\alpha}\right)_{i_{\alpha} j_{\alpha}} e_{i_{1}} \otimes \cdots \otimes e_{i_{r}}=\sum_{i \in I} m_{i, j} e_{i} .
\end{aligned}
$$

(ii) For all $\sigma \in G$ and $i, j \in I$, we have

$$
\begin{aligned}
(m \sigma)_{i, j} & =\prod_{\alpha=1}^{r}\left(m_{\sigma(\alpha)}\right)_{i_{\alpha} j_{\alpha}}=\prod_{\alpha}\left(m_{\alpha}\right)_{i_{\sigma^{-1}(\alpha)} j_{\sigma^{-1}(\alpha)}} \\
& =\prod_{\alpha}\left(m_{\alpha}\right)_{\left(i \sigma^{-1}\right)_{\alpha}\left(j \sigma^{-1}\right)_{\alpha}} \\
& =m_{i \sigma^{-1}, j \sigma^{-1}}
\end{aligned}
$$


Let $\Gamma=\mathrm{GL}_{n}(K) \subseteq M$ and put $\Gamma^{r}=\Gamma \times \cdots \times \Gamma \subseteq M^{r}$. The right action of $G$ on $M^{r}$ induces an action on the group algebra $K \Gamma^{r}$ of $\Gamma^{r}$ over $K$. Denote by $R$ the subalgebra of $K \Gamma^{r}$ consisting of those elements fixed by $G$ :

$$
R=\left(K \Gamma^{r}\right)^{G}=\left\{\kappa \in K \Gamma^{r} \mid \kappa \sigma=\kappa \text { for all } \sigma \in G\right\} .
$$

The set $\left\{\bar{g} \mid g \in \Gamma^{r}\right\}$ is a $K$-basis for $R$, where

$$
\bar{g}=\frac{1}{|G|} \sum_{\sigma \in G} g \sigma .
$$

Let $V$ be an $R$-module with finite $K$-basis $\left\{v_{j} \mid j \in J\right\}$. For $\kappa \in R$ and $j \in J$, we have

$$
\kappa v_{j}=\sum_{i \in J} \alpha_{i j}(\kappa) v_{i}
$$

for some $\alpha_{i j} \in R^{*}=\operatorname{Hom}_{K}(R, K)$ (dual space of $R$ ). The $K$-linear span of the set $\left\{\alpha_{i j} \mid i, j \in J\right\}$ is the coefficient space of the $R$-module $V$, denoted $\operatorname{cf}(V)$. This space is independent of the choice of basis for $V$.

If $R_{K}(R)$ is the representative $K$-bialgebra of the multiplicative semigroup $R$, then $V$ is a right $R_{K}(R)$-comodule with structure map $\psi: V \rightarrow V \otimes$ $R_{K}(R)$ given by $\psi\left(v_{j}\right)=\sum_{i \in J} v_{i} \otimes \alpha_{i j}$. We have

$$
\begin{gathered}
\triangle\left(\alpha_{i j}\right)=\sum_{k} \alpha_{i k} \otimes \alpha_{k j}, \\
\epsilon\left(\alpha_{i j}\right)=\delta_{i j},
\end{gathered}
$$

so that $\operatorname{cf}(V)$ is a subcoalgebra of $R_{K}(R)$ [Ab, p. 125].

Let $\rho: R \rightarrow \operatorname{End}_{K}(V)$ be the representation afforded by the $R$-module $V$.

\subsection{LEMMA.}

(i) For $\kappa \in R$, we have $\kappa \in \operatorname{ker} \rho$ if and only if $f(\kappa)=0$ for all $f \in$ $\operatorname{cf}(V)$.

(ii) For $f \in R^{*}$, we have $f \in \operatorname{cf}(V)$ if and only if $f(\kappa)=0$ for all $\kappa \in \operatorname{ker} \rho$.

Proof. See proof of [Ma, 2.2.1].

1.3 Theorem. The map $\psi: \operatorname{im} \rho \rightarrow \operatorname{cf}(V)^{*}$ given by $\psi(\rho(\kappa))(c)=c(\kappa)$ is a K-isomorphism.

Proof. The bilinear map $\operatorname{im} \rho \times \operatorname{cf}(V) \rightarrow K$ given by $\langle\rho(\kappa), c\rangle=c(\kappa)$ is well defined and nondegenerate by Lemma 1.2. Since the spaces im $\rho$ and $\operatorname{cf}(V)$ are finite dimensional, the induced map $\psi: \operatorname{im} \rho \rightarrow \operatorname{cf}(V)^{*}$ is a $K$ isomorphism. 


\section{Generalized coefficient CoAlgebra And Schur Algebra}

We continue to assume that $K$ is an infinite field of characteristic not a divisor of $|G|$. Recall that $R$ is the subalgebra of $K \Gamma^{r}$ consisting of those elements fixed by $G$.

For $i, j \in I$ define $c_{i, j}: R \rightarrow K$ by putting

$$
c_{i, j}(\bar{g})=\frac{1}{|G|} \sum_{\sigma \in G}(g \sigma)_{i, j}
$$

$\left(g \in \Gamma^{r}\right)$ and extending linearly to $R$.

2.1 Lemma. The function $c_{i, j}$ is well-defined.

Proof. Let $g, h \in \Gamma^{r}$ and assume that $\bar{g}=\bar{h}$. Then $h=g \tau$ for some $\tau \in G$, so

$$
c_{i, j}(\bar{h})=\frac{1}{|G|} \sum_{\sigma \in G}(h \sigma)_{i, j}=\frac{1}{|G|} \sum_{\sigma \in G}(g \tau \sigma)_{i, j}=c_{i, j}(\bar{g}) .
$$

2.2 Lemma. For $\kappa \in R$ and $j \in I$,

$$
\kappa e_{j}=\sum_{i \in I} c_{i, j}(\kappa) e_{i}
$$

Proof. Let $g \in \Gamma^{r}$. It suffices to establish the equality in the case $\kappa=\bar{g}$. For $j \in I$ we have

$$
\bar{g} e_{j}=\frac{1}{|G|} \sum_{\sigma \in G}(g \sigma) e_{j}=\frac{1}{|G|} \sum_{\sigma \in G} \sum_{i \in I}(g \sigma)_{i, j} e_{i}=\sum_{i \in I} c_{i, j}(\bar{g}) e_{i},
$$

where the second equality uses Lemma 1.1.

The group $G$ acts on $I$ from the right by $i \sigma=\left(i_{\sigma(1)}, i_{\sigma(2)}, \ldots, i_{\sigma(r)}\right)$. In turn, $G$ acts on $I \times I$ diagonally: $(i, j) \sigma=(i \sigma, j \sigma)$. For $i, j, k, l \in I$, put $(i, j) \sim(k, l)$ if $(k, l)=(i, j) \sigma=(i \sigma, j \sigma)$ for some $\sigma \in G$.

2.3 Lemma. $c_{i, j}=c_{k, l}$ if and only if $(i, j) \sim(k, l)$.

Proof. For $i, j \in I$, define $\hat{c}_{i, j}: M^{r} \rightarrow K$ by

$$
\hat{c}_{i, j}(m)=\frac{1}{|G|} \sum_{\sigma \in G}(m \sigma)_{i, j}
$$

and note that for $g \in \Gamma^{r}$ we have $\hat{c}_{i, j}(g)=c_{i, j}(\bar{g})$.

Let $i, j, k, l \in I$ and assume that $c_{i, j}=c_{k, l}$. Then $\hat{c}_{i, j}(g)=\hat{c}_{k, l}(g)$ for each $g \in \Gamma^{r}$ and, since $\Gamma^{r}$ is Zariski dense in $M^{r}$, it follows that $\hat{c}_{i, j}=\hat{c}_{k, l}$.

Define $b_{i, j}=\left(b_{i_{1} j_{1}}, \ldots, b_{i_{r} j_{r}}\right) \in M^{r}$, where $\left(b_{a b}\right)_{c d}=\delta_{(a, b),(c, d)}$ (Kronecker delta) and note that $\left(b_{i, j}\right)_{x, y}=\delta_{(i, j),(x, y)}$. For $x, y \in I$, we get, using Lemma 
1.1

$$
\begin{aligned}
\hat{c}_{x, y}\left(b_{i, j}\right) & =\frac{1}{|G|} \sum_{\sigma \in G}\left(b_{i, j} \sigma\right)_{x, y} \\
& =\frac{1}{|G|} \sum_{\sigma \in G}\left(b_{i, j}\right)_{x \sigma^{-1}, y \sigma^{-1}} \\
& = \begin{cases}\frac{\left|G_{(i, j)}\right|}{|G|}, & (x, y) \sim(i, j) \\
0, & \text { otherwise, }\end{cases}
\end{aligned}
$$

where $G_{(i, j)}$ is the stabilizer of $(i, j)$ in $G$. Since

$$
\hat{c}_{k, l}\left(b_{i, j}\right)=\hat{c}_{i, j}\left(b_{i, j}\right)=\frac{\left|G_{(i, j)}\right|}{|G|} \neq 0,
$$

we conclude that $(k, l) \sim(i, j)$.

Now assume that $(i, j) \sim(k, l)$ so that $(k, l)=(i \sigma, j \sigma)$ for some $\sigma \in G$. For any $g \in \Gamma^{r}$, Lemma 1.1 gives

$$
\left(g \sigma^{-1}\right)_{i, j}=g_{i \sigma, j \sigma}=g_{k, l}
$$

So

$$
\begin{aligned}
c_{i, j}(\bar{g}) & =\frac{1}{|G|} \sum_{\tau \in G}(g \tau)_{i, j}=\frac{1}{|G|} \sum_{\tau \in G}\left(g \tau \sigma^{-1}\right)_{i, j} \\
& =\frac{1}{|G|} \sum_{\tau \in G}(g \tau)_{k, l}=c_{k, l}(\bar{g}) .
\end{aligned}
$$

Therefore, $c_{i, j}=c_{k, l}$.

Let $A$ be the coefficient space of the $R$-module $E^{\otimes r}$.

\subsection{Theorem.}

(i) The space $A$ has $K$-basis $\left\{c_{i, j} \mid(i, j) \in B\right\}$, where $B$ is a set of representatives for the orbits of $I \times I$ under the diagonal action of $G$.

(ii) $A$ is a coalgebra with structure maps

$$
\begin{gathered}
\triangle\left(c_{i, j}\right)=\sum_{k \in I} c_{i, k} \otimes c_{k, j}, \\
\epsilon\left(c_{i, j}\right)=\delta_{i, j} .
\end{gathered}
$$

Proof. (i) By Lemma 2.2, the space $A$ is the $K$-linear span of $\left\{c_{i, j} \mid i, j \in I\right\}$, which equals $\left\{c_{i, j} \mid(i, j) \in B\right\}$ by Lemma 2.3.

Suppose that $\sum_{(i, j) \in B} \alpha_{i, j} c_{i, j}=0$ with $\alpha_{i, j} \in K$. Let $\hat{c}_{i, j}: M^{r} \rightarrow$ $K$ be as in the proof of Lemma 2.3. Since $\hat{c}_{i, j}(g)=c_{i, j}(\bar{g})$, we have $\sum_{(i, j) \in B} \alpha_{i, j} \hat{c}_{i, j}(g)=0$ for all $g \in \Gamma^{r}$. Since $\Gamma^{r}$ is Zariski dense in $M^{r}$, 
we have $\sum_{(i, j) \in B} \alpha_{i, j} \hat{c}_{i, j}=0$. Let $(x, y) \in B$. With $b_{x, y}$ as in the proof of Lemma 2.3 we have (using the formula in that proof)

$$
\alpha_{x, y}=\frac{|G|}{\left|G_{(x, y)}\right|} \sum_{(i, j) \in B} \alpha_{i, j} \hat{c}_{i, j}\left(b_{x, y}\right)=0 .
$$

Therefore, $\left\{c_{i, j} \mid(i, j) \in B\right\}$ is linearly independent.

(ii) This follows from Lemma 2.2 and the discussion in Section 1.

The group $G$ acts on $E^{\otimes r}$ by $e_{i} \sigma=e_{i \sigma}(i \in I)$ and this action extends linearly to make $E^{\otimes r}$ a right $K G$-module. In fact $E^{\otimes r}$ is a $\left(K M^{r}, K G\right)$ bimodule. One checks that $v \sigma=v_{\sigma(1)} \otimes \cdots \otimes v_{\sigma(r)}$ for all $v=v_{1} \otimes \cdots \otimes v_{r} \in$ $E^{\otimes r}$.

The set $N=\operatorname{End}_{K}\left(E^{\otimes r}\right)$ is a right $K G$-module with action given by $(f \sigma)(v)=f\left(v \sigma^{-1}\right) \sigma(\sigma \in G)$. Moreover, $N^{G}=\operatorname{End}_{K G}\left(E^{\otimes r}\right)$, where $N^{G}$ is the set of those elements of $N$ fixed by $G$.

Let $\hat{T}: K \Gamma^{r} \rightarrow \operatorname{End}_{K}\left(E^{\otimes r}\right)$ be the representation afforded by the $K \Gamma^{r}$ module $E^{\otimes r}$.

2.5 LEMMA. $\hat{T}$ is a KG-homomorphism.

Proof. Let $g \in \Gamma^{r}, \sigma \in G$, and $v=v_{1} \otimes \cdots \otimes v_{r} \in E^{\otimes r}$. We have

$$
\begin{aligned}
{[\hat{T}(g) \sigma](v) } & =\left(\hat{T}(g)\left(v \sigma^{-1}\right)\right) \sigma \\
& =\left(\hat{T}(g)\left(v_{\sigma^{-1}(1)} \otimes \cdots \otimes v_{\sigma^{-1}(r)}\right) \sigma\right. \\
& =\left(g_{1} v_{\sigma^{-1}(1)} \otimes \cdots \otimes g_{r} v_{\sigma^{-1}(r)}\right) \sigma \\
& =w_{\sigma(1)} \otimes \cdots \otimes w_{\sigma(r)} \quad\left(w_{i}:=g_{i} v_{\sigma^{-1}(i)}\right) \\
& =g_{\sigma(1)} v_{1} \otimes \cdots \otimes g_{\sigma(r)} v_{r} \\
& =[\hat{T}(g \sigma)](v) .
\end{aligned}
$$

Therefore, $\hat{T}(g \sigma)=\hat{T}(g) \sigma$ as claimed.

The $K$-space $N$ has basis $\left\{e_{i, j} \mid i, j \in I\right\}$, where

$$
e_{i, j}\left(e_{k}\right)=\delta_{j k} e_{i}
$$

(Kronecker delta).

The map $T=\left.\hat{T}\right|_{R}: R \rightarrow \operatorname{End}_{K}\left(E^{\otimes r}\right)$ is the representation afforded by the $R$-module $E^{\otimes r}$.

2.6 Lemma. For $\kappa \in R$,

$$
T(\kappa)=\sum_{i, j \in I} c_{i, j}(\kappa) e_{i, j}
$$


Proof. Let $\kappa \in R$. Since $\left\{e_{i, j} \mid i, j \in I\right\}$ is a basis for $N$, we have $T(\kappa)=$ $\sum_{i, j \in I} \alpha_{i, j} e_{i, j}$ for some $\alpha_{i, j} \in K$. For each $j \in I$ we have

$$
\begin{aligned}
\sum_{i \in I} \alpha_{i, j} e_{i} & =\sum_{i, l \in I} \alpha_{i, l} e_{i, l}\left(e_{j}\right) \\
& =T(\kappa)\left(e_{j}\right)=\kappa e_{j} \\
& =\sum_{i \in I} c_{i, j}(\kappa) e_{i} \quad(\text { Lemma 2.2). }
\end{aligned}
$$

Therefore, $\alpha_{i, j}=c_{i, j}(\kappa)$ for all $i, j \in I$ and the claim follows.

Put $S=\operatorname{im} T$.

2.7 Theorem. $S=\operatorname{End}_{K G}\left(E^{\otimes r}\right)$.

Proof. Since $R=\left(K \Gamma^{r}\right)^{G}$, it follows from Lemma 2.5 that $S=\operatorname{im} T=$ $\left.\operatorname{im} \hat{T}\right|_{R} \subseteq N^{G}=\operatorname{End}_{K G}\left(E^{\otimes r}\right)$.

For the other inclusion, it is enough to show that the orthogonal complement of $S$ in $N^{G}$ is trivial, where $N$ has the (non-degenerate) bilinear form induced by $\left\langle e_{i, j}, e_{k, l}\right\rangle=\delta_{(i, j),(k, l)}$ (Kronecker delta). Let $f=\sum_{i, j \in I} \alpha_{i, j} e_{i, j}$ be an element of this orthogonal complement. For $\kappa \in R$, we have

$$
\begin{aligned}
0 & =\langle f, T(\kappa)\rangle \\
& =\sum_{i, j \in I} \sum_{k, l \in I} \alpha_{i, j} c_{k, l}(\kappa)\left\langle e_{i, j}, e_{k, l}\right\rangle \\
& =\sum_{i, j \in I} \alpha_{i, j} c_{i, j}(\kappa) .
\end{aligned}
$$

Since $f$ is fixed by $G$, it follows that $\alpha_{i \sigma, j \sigma}=\alpha_{i, j}$ for every $\sigma \in G$. Therefore, choosing a set $B$ of orbit representatives of $I \times I$ under the diagonal action of $G$, we have

$$
0=\sum_{i, j \in I} \alpha_{i, j} c_{i, j}=\sum_{(i, j) \in B} \sum_{\sigma \in G / G_{(i, j)}} \alpha_{i \sigma, j \sigma} c_{i \sigma, j \sigma}=\sum_{(i, j) \in B}\left|G: G_{(i, j)}\right| \alpha_{i, j} c_{i, j},
$$

where $G_{(i, j)}$ is the stabilizer of $(i, j)$ in $G$ and $G / G_{(i, j)}$ is a set of representatives for the right cosets of $G_{(i, j)}$ in $G$, and where we have used Lemma 2.3. Using Theorem 2.4, we have $\alpha_{i, j}=0$ for each $(i, j) \in B$ (and hence for each $i, j \in I$ ) so that $f=0$ as desired.

For a $K$-coalgebra $C$ the dual space $C^{*}$ has a natural structure of $K$ algebra $\left[\mathrm{Ab}\right.$, p. 55]. In particular, $A^{*}$ is a $K$-algebra.

2.8 Theorem. The map $\psi: S \rightarrow A^{*}$ given by $\psi(T(\kappa))(c)=c(\kappa)$ is an isomorphism of $K$-algebras.

Proof. By Theorem 1.3, $\psi$ is a $K$-isomorphism. Using the argument of [Ma, 2.3.5 and following paragraph] with the aid of Lemma 2.6, one sees that it is an algebra homomorphism as well. 
Assume for the moment that $G=\Sigma_{r}$ (full symmetric group). The diagonal subgroup $D=\{(a, \ldots, a) \mid a \in \Gamma\}$ of $\Gamma^{r}$ identifies naturally with $\Gamma$. Note that $K D \subseteq R$. The functions $\left.c_{i, j}\right|_{K D}(i, j \in I)$ coincide with the standard basis vectors of the classical coefficient coalgebra $A_{r}[\mathrm{Ma}, 1.3 .4]$. In view of Theorem 2.4, the map $\left.c_{i, j} \mapsto c_{i, j}\right|_{K D}$ defines a coalgebra isomorphism $A \cong A_{r}$.

Next, the image of the map $\left.T\right|_{K D}: K D \rightarrow \operatorname{End}_{K}\left(E^{\otimes r}\right)$ is the classical Schur algebra $S_{r}$ [Ma, 2.1.1]. In particular, $S_{r} \subseteq S$. Since $S \cong A^{*}$ (Theorem 2.8) and $S_{r} \cong A_{r}^{*}$ [Ma, 2.3.5] we have $S=S_{r}$. (One could also see this by using Theorem 2.7 and Schur's Commutation Theorem [Ma, 2.1.3].)

In the other extreme, if $G=\{e\}$, then $S=\operatorname{End}_{K}\left(E^{\otimes r}\right)$ (Theorem 2.7).

\section{Decompositions by irreducible Characters}

From now on we let $K$ be the field $\mathbf{C}$ of complex numbers and adjust the notation accordingly. Let $\operatorname{Irr}(G)$ be the set of (ordinary) irreducible characters of $G$ and fix $\chi \in \operatorname{Irr}(G)$. Define a linear map $\tau_{\chi}: A \rightarrow A$ by

$$
\tau_{\chi}\left(c_{i, j}\right)=\frac{\chi(e)}{|G|} \sum_{\sigma \in G} \chi\left(\sigma^{-1}\right) c_{i, j \sigma}
$$

3.1 Theorem. The function $\tau_{\chi}$ is well-defined and

$$
\left(\tau_{\chi} \otimes \tau_{\chi}\right) \circ \triangle=\triangle \circ \tau_{\chi}
$$

Proof. Suppose that $c_{i, j}=c_{k, l}$. By Lemma 2.3, we have $(k, l)=(i \sigma, j \sigma)$ for some $\sigma \in G$. Then

$$
\begin{aligned}
\sum_{\mu \in G} \chi\left(\mu^{-1}\right) c_{k, l \mu} & =\sum_{\mu \in G} \chi\left(\mu^{-1}\right) c_{i \sigma, j \sigma \mu}=\sum_{\mu \in G} \chi\left(\mu^{-1}\right) c_{i, j \sigma \mu \sigma^{-1}} \\
& =\sum_{\mu \in G} \chi\left(\sigma^{-1} \mu^{-1} \sigma\right) c_{i, j \mu}=\sum_{\mu \in G} \chi\left(\mu^{-1}\right) c_{i, j \mu}
\end{aligned}
$$

so $\tau_{\chi}\left(c_{k, l}\right)=\tau_{\chi}\left(c_{i, j}\right)$ and $\tau_{\chi}$ is well-defined.

Let $i, j \in I$. Using Theorem 2.4 we have

$$
\begin{gathered}
{\left[\left(\tau_{\chi} \otimes \tau_{\chi}\right) \circ \triangle\right]\left(c_{i, j}\right)=\left(\tau_{\chi} \otimes \tau_{\chi}\right)\left(\sum_{k \in I} c_{i, k} \otimes c_{k, j}\right)=\sum_{k} \tau_{\chi}\left(c_{i, k}\right) \otimes \tau_{\chi}\left(c_{k, j}\right)} \\
=\sum_{k}\left(\frac{\chi(e)}{|G|} \sum_{\sigma \in G} \chi\left(\sigma^{-1}\right) c_{i, k \sigma}\right) \otimes\left(\frac{\chi(e)}{|G|} \sum_{\mu \in G} \chi\left(\mu^{-1}\right) c_{k, j \mu}\right) \\
=\frac{\chi(e)^{2}}{|G|^{2}} \sum_{\sigma, \mu} \chi\left(\sigma^{-1}\right) \chi\left(\mu^{-1}\right)\left(\sum_{k} c_{i \sigma^{-1}, k} \otimes c_{k, j \mu}\right) \\
=\frac{\chi(e)^{2}}{|G|^{2}} \sum_{\sigma, \mu} \chi\left(\sigma^{-1}\right) \chi\left(\mu^{-1}\right) \triangle\left(c_{i \sigma^{-1}, j \mu}\right) .
\end{gathered}
$$


Since $c_{i \sigma^{-1}, j \mu}=c_{i, j \mu \sigma}$, we can put $\rho=\mu \sigma$ on the right and get

$$
\begin{aligned}
{\left[\left(\tau_{\chi} \otimes \tau_{\chi}\right) \circ \triangle\right]\left(c_{i, j}\right) } & =\triangle\left[\frac{\chi(e)}{|G|} \sum_{\rho}\left(\frac{\chi(e)}{|G|} \sum_{\sigma} \chi\left(\sigma^{-1}\right) \chi\left(\sigma \rho^{-1}\right)\right) c_{i, j \rho}\right] \\
& =\triangle\left[\frac{\chi(e)}{|G|} \sum_{\rho} \chi\left(\rho^{-1}\right) c_{i, j \rho}\right]=\left[\triangle \circ \tau_{\chi}\right]\left(c_{i, j}\right),
\end{aligned}
$$

where we have used the generalized orthogonality relation [Is, 2.13] in the next to the last step. The theorem follows.

Define

$$
t_{\chi}=\frac{\chi(e)}{|G|} \sum_{\sigma \in G} \chi\left(\sigma^{-1}\right) \sigma \in \mathbf{C} G .
$$

We have $t_{\chi}^{2}=t_{\chi}$ [Is, 2.12 and proof of 2.13]. Put $E^{\chi}=E^{\otimes r} t_{\chi}$, the symmetrized tensor space corresponding to the character $\chi$.

Let $A_{\chi}$ denote the coefficient coalgebra of the $R$-module $E^{\chi}$ and let $T_{\chi}$ : $R \rightarrow \operatorname{End}_{\mathbf{C}}\left(E^{\chi}\right)$ be the representation afforded by $E^{\chi}$. Put $S_{\chi}=\operatorname{im} T_{\chi}$.

The space $N=\operatorname{End}_{\mathbf{C}}\left(E^{\otimes r}\right)$ is a left $\mathbf{C} G$-module with action determined by $(\sigma f)(v)=f(v \sigma)(\sigma \in G)$.

\subsection{TheOREM.}

(i) $t_{\chi} S$ is a subalgebra of $S$ and $S_{\chi} \cong t_{\chi} S$ as $\mathbf{C}$-algebras,

(ii) $\tau_{\chi}(A)$ is a subcoalgebra of $A$ and $A_{\chi}=\tau_{\chi}(A)$.

Proof. (i) For $f, g, \in S$ and $v \in E^{\otimes r}$, we have, using Theorem 2.7,

$$
\begin{aligned}
{\left[\left(t_{\chi} f\right)\left(t_{\chi} g\right)\right](v) } & =\left(t_{\chi} f\right)\left(g\left(v t_{\chi}\right)\right)=f\left(g\left(v t_{\chi}\right) t_{\chi}\right) \\
& =f\left(g\left(v t_{\chi}^{2}\right)\right)=f\left(g\left(v t_{\chi}\right)\right)=(f g)\left(v t_{\chi}\right) \\
& =\left[t_{\chi}(f g)\right](v) .
\end{aligned}
$$

Therefore, $\left(t_{\chi} f\right)\left(t_{\chi} g\right)=t_{\chi}(f g)$, so that $t_{\chi} S$ is a subalgebra of $S$.

Define $\varphi: t_{\chi} S \rightarrow S_{\chi}$ by $\varphi\left(t_{\chi} T(\kappa)\right)=T_{\chi}(\kappa)(\kappa \in R)$. We have

$$
\begin{aligned}
t_{\chi} T(\kappa)=t_{\chi} T(\lambda) & \Longleftrightarrow\left(t_{\chi} T(\kappa)\right)(v)=\left(t_{\chi} T(\lambda)\right)(v) \quad \text { for all } v \in E^{\otimes r} \\
& \Longleftrightarrow T(\kappa)\left(v t_{\chi}\right)=T(\lambda)\left(v t_{\chi}\right) \text { for all } v \in E^{\otimes r} \\
& \Longleftrightarrow T_{\chi}(\kappa)=T_{\chi}(\lambda),
\end{aligned}
$$

so $\varphi$ is well defined and injective. It is immediate that $\varphi$ is surjective and $\mathbf{C}$-linear, so it is a $\mathbf{C}$-isomorphism.

Finally, for $\kappa, \lambda \in R$, we have, using the first part of the proof,

$$
\begin{aligned}
\varphi\left(\left(t_{\chi} T(\kappa)\right)\left(t_{\chi} T(\lambda)\right)\right. & =\varphi\left(t_{\chi} T(\kappa) T(\lambda)\right)=\varphi\left(t_{\chi} T(\kappa \lambda)\right) \\
& =T_{\chi}(\kappa \lambda)=T_{\chi}(\kappa) T_{\chi}(\lambda)=\varphi\left(t_{\chi} T(\kappa)\right) \varphi\left(t_{\chi} T(\lambda)\right),
\end{aligned}
$$

so $\varphi$ is a $\mathbf{C}$-algebra isomorphism.

(ii) First, $\tau_{\chi}(A)$ is a subcoalgebra of $A$ by Theorem 3.1. 
Next, we show that $\tau_{\chi}(A) \subseteq A_{\chi}$. By Lemma 1.2(ii), it is enough to show that $\tau_{\chi}\left(c_{i, j}\right)(\kappa)=0$ for all $\kappa \in \operatorname{ker} T_{\chi}$ and $i, j \in I$. Let $\kappa \in \operatorname{ker} T_{\chi}$ and fix $i, j \in I$.

The group $G$ acts on the set $N^{R}$ of functions from $R$ to $N$ from the left by $(\sigma f)(\mu)=\sigma f(\mu)$ and on $N^{*}$ from the right by $(f \sigma)(e)=f(\sigma e)$.

(Step 1) $e_{i, j}^{*} \circ t_{\chi} T=e_{i, j}^{*} t_{\chi} \circ T$. We have, for $\mu \in R$,

$$
\left(e_{i, j}^{*} \circ t_{\chi} T\right)(\mu)=e_{i, j}^{*}\left(t_{\chi} T(\mu)\right)=\left(e_{i, j}^{*} t_{\chi}\right)(T(\mu))=\left(e_{i, j}^{*} t_{\chi} \circ T\right)(\mu) .
$$

(Step 2) $\tau_{\chi}\left(c_{i, j}\right)=e_{i, j}^{*} \circ t_{\chi} T$. One checks that, for $\sigma \in G, \sigma e_{i, j}=e_{i, j \sigma^{-1}}$ and, in turn, $e_{i, j}^{*} \sigma=e_{i, j \sigma}^{*}$. We have

$$
\begin{aligned}
\tau_{\chi}\left(c_{i, j}\right) & =\frac{\chi(e)}{|G|} \sum_{\sigma \in G} \chi\left(\sigma^{-1}\right) c_{i, j \sigma} \\
& =\frac{\chi(e)}{|G|} \sum_{\sigma} \chi\left(\sigma^{-1}\right) e_{i, j \sigma}^{*} \circ T \\
& =e_{i, j}^{*} t_{\chi} \circ T \\
& =e_{i, j}^{*} \circ t_{\chi} T
\end{aligned}
$$

(Step 3) $\left(t_{\chi} T\right)(\kappa)=0$. For $v \in E^{\otimes r}$ we have

$$
\left(\left(t_{\chi} T\right)(\kappa)\right)(v)=\left(t_{\chi} T(\kappa)\right)(v)=T(\kappa)\left(v t_{\chi}\right)=\kappa\left(v t_{\chi}\right)=0
$$

since $v t_{\chi} \in E^{\chi}$ and $\kappa \in \operatorname{ker} T_{\chi}$.

Therefore,

$$
\begin{aligned}
\tau_{\chi}\left(c_{i, j}\right)(\kappa) & =e_{i, j}^{*}\left(\left(t_{\chi} T\right)(\kappa)\right) \\
& =e_{i, j}^{*}(0) \\
& =0,
\end{aligned}
$$

and we conclude that $\tau_{\chi}(A) \subseteq A_{\chi}$.

Finally, we show that $A_{\chi} \subseteq \tau_{\chi}(A)$. For $\kappa \in R$ and $j \in I$ we have

$$
\begin{aligned}
\kappa e_{j} t_{\chi} & =\kappa e_{j} t_{\chi}^{2}=\frac{\chi(e)}{|G|} \sum_{\sigma \in G} \chi\left(\sigma^{-1}\right) \kappa e_{j \sigma} t_{\chi} \\
& =\frac{\chi(e)}{|G|} \sum_{\sigma} \chi\left(\sigma^{-1}\right) \sum_{i \in I} c_{i, j \sigma}(\kappa) e_{i} t_{\chi} \\
& =\sum_{i} \tau_{\chi}\left(c_{i, j}\right)(\kappa) e_{i} t_{\chi}
\end{aligned}
$$

and, since some subset of $\left\{e_{i} t_{\chi} \mid i \in I\right\}$ is a basis for $E^{\chi}$, the claim follows.

It follows from the orthogonality relations of characters [Is, 2.13, 2.18] that the maps $\tau_{\chi}(\chi \in \operatorname{Irr}(G))$ are pairwise orthogonal idempotents that sum to $1_{A}$ :

(i) $\tau_{\chi} \tau_{\psi}=\delta_{\chi, \psi} \tau_{\chi}($ Kronecker delta),

(ii) $\sum_{\chi \in \operatorname{Irr}(G)} \tau_{\chi}=1_{A}$. 
3.3 Theorem. $A \cong \bigoplus_{\chi \in \operatorname{Irr}(G)} A_{\chi}$.

Proof. By (ii) of the preceding paragraph and Theorem 3.2(ii),

$$
A=\sum_{\chi \in \operatorname{Irr}(G)} \tau_{\chi}(A)=\sum_{\chi \in \operatorname{Irr}(G)} A_{\chi}
$$

and by (i) of the preceding paragraph the sum is direct.

3.4 Theorem. The map $\psi_{\chi}: S_{\chi} \rightarrow A_{\chi}^{*}$ given by $\psi_{\chi}\left(T_{\chi}(\kappa)\right)(c)=c(\kappa)$ is a C-algebra isomorphism.

Proof. Let $\psi: S \rightarrow A^{*}$ be the isomorphism of Theorem 2.8 and let $\eta: S \rightarrow$ $S_{\chi}$ be the epimorphism induced by restriction: $\eta(T(\kappa))=\left.T(\kappa)\right|_{E \chi}$. Then $\psi(\operatorname{ker} \eta)=A_{\chi}^{0}\left(=\right.$ annihilator of $\left.A_{\chi}\right)$. Indeed, for $\kappa \in R$, we have

$$
\begin{aligned}
\psi(T(\kappa)) \in A_{\chi}^{0} & \Longleftrightarrow \psi(T(\kappa))(c)=0 \quad \forall c \in A_{\chi} \\
& \Longleftrightarrow c(\kappa)=0 \quad \forall c \in A_{\chi} \\
& \Longleftrightarrow \kappa \in \operatorname{ker} T_{\chi} \quad(\text { Lemma 1.2(i)) } \\
& \left.\Longleftrightarrow T(\kappa)\right|_{E \chi}=T_{\chi}(\kappa)=0 \\
& \Longleftrightarrow T(\kappa) \in \operatorname{ker} \eta .
\end{aligned}
$$

Therefore, we have $\mathbf{C}$-algebra isomorphisms

$$
S_{\chi} \cong S / \operatorname{ker} \eta \cong A^{*} / A_{\chi}^{0} \cong A_{\chi}^{*}
$$

(the last $\mathbf{C}$-isomorphism is an algebra isomorphism by Theorem 3.2(ii) and [Ab, 2.3.1(ii)]). Calling the composition $\psi_{\chi}$ and the composition of just the last two $\varphi$, we have

$$
\psi_{\chi}\left(T_{\chi}(\kappa)\right)(c)=\varphi(\overline{T(\kappa)})(c)=\overline{\psi(T(\kappa))}(c)=\psi(T(\kappa))(c)=c(\kappa) .
$$

3.5 ThEOREM. $S \cong \bigoplus_{\chi \in \operatorname{Irr}(G)} S_{\chi}$.

Proof. This follows from Theorems 2.8, 3.3, and 3.4.

For $i \in I$, let $G_{i}$ denote the stabilizer of $i$ in $G$.

3.6 Theorem. We have

$$
\operatorname{dim}_{\mathbf{C}} A_{\chi}=\frac{\chi(e)}{|G|} \sum_{(i, j) \in B} \sum_{\sigma \in G_{j} G_{i}} \chi\left(\sigma^{-1}\right),
$$

where $B$ is a set of representatives for the orbits of $I \times I$ under the diagonal action of $G$.

Proof. By Theorem 3.2(ii), we have $A_{\chi}=\tau_{\chi}(A)$. Since $\tau_{\chi}^{2}=\tau_{\chi}$, an eigenvalue of $\tau_{\chi}$ is either 1 or 0 , so the rank of $\tau_{\chi}$ equals its trace. Therefore, it is enough to show that the trace of $\tau_{\chi}$ is given by the formula on the right. 
Fix $i, j \in I$ and let $D$ be a set of representatives of the $\left(G_{j}, G_{i}\right)$ double cosets in $G$ chosen with $e \in D$. For $\delta \in D$, let $R_{\delta}$ be a set of representatives of the right cosets of $G_{i} \cap \delta^{-1} G_{j} \delta$ in $G_{i}$ so that

$$
G_{j} \delta G_{i}=\bigcup_{\rho \in R_{\delta}} G_{j} \delta \rho
$$

(disjoint union) [Su, proof of 3.8(iv)]. We have

$$
\begin{aligned}
\tau_{\chi}\left(c_{i, j}\right) & =\frac{\chi(e)}{|G|} \sum_{\sigma \in G} \chi\left(\sigma^{-1}\right) c_{i, j \sigma} \\
& =\frac{\chi(e)}{|G|} \sum_{\substack { \delta \in D \\
\begin{subarray}{c}{\mu \in G_{j} \\
\rho \in R_{\delta}{ \delta \in D \\
\begin{subarray} { c } { \mu \in G _ { j } \\
\rho \in R _ { \delta } } }\end{subarray}} \chi\left((\mu \delta \rho)^{-1}\right) c_{i, j(\mu \delta \rho) .}
\end{aligned}
$$

In the last sum, we have

$$
c_{i, j(\mu \delta \rho)}=c_{i, j(\delta \rho)}=c_{i \rho^{-1}, j \delta}=c_{i, j \delta}
$$

so

$$
\tau_{\chi}\left(c_{i, j}\right)=\frac{\chi(e)}{|G|} \sum_{\delta \in D} \sum_{\substack{\mu \in G_{j} \\ \rho \in R_{\delta}}} \chi\left((\mu \delta \rho)^{-1}\right) c_{i, j \delta} .
$$

Let $\delta, \epsilon \in D$ and assume that $(i, j \delta) \sim(i, j \epsilon)$ so that $(i, j \delta)=(i \pi, j \epsilon \pi)$ for some $\pi \in G$. Then $\pi \in G_{i}$ and $\epsilon \pi \delta^{-1} \in G_{j}$, whence $G_{j} \epsilon G_{i}=G_{j} \epsilon \pi G_{i}=$ $G_{j} \delta G_{i}$, implying that $\epsilon=\delta$. We conclude that the $c_{i, j \delta}$ appearing in the linear combination above are distinct and that $c_{i, j \delta}=c_{i, j}$ if and only if $\delta=e$. Therefore,

$$
\begin{aligned}
\operatorname{tr} \tau_{\chi} & =\sum_{(i, j) \in B}\left(\frac{\chi(e)}{|G|} \sum_{\substack{\mu \in G_{j} \\
\rho \in R_{e}}} \chi\left((\mu e \rho)^{-1}\right)\right) \\
& =\frac{\chi(e)}{|G|} \sum_{(i, j) \in B} \sum_{\sigma \in G_{j} G_{i}} \chi\left(\sigma^{-1}\right)
\end{aligned}
$$

as claimed.

\section{REFERENCES}

[Ab] E. Abe, Hopf algebras, Cambridge Univ. Press, Cambridge, 1976.

[Is] I. M. Isaacs, Character theory of finite groups, Dover, New York, 1976.

[Ma] S. Martin, Schur algebras and representation theory, Cambridge Univ. Press, Cambridge, 1993.

[Su] M. Suzuki, Group Theory I, Springer-Verlag, Berlin, 1982.

$[\mathrm{Tu}]$ D. P. Turner, "Coefficient space properties and a Schur algebra generalization," Ph.D. dissertation, Auburn University, Auburn, Alabama, 2005.

Randall R. Holmes, Department of Mathematics and Statistics, Auburn University, Auburn AL, 36849, holmerr@auburn.edu

David P. Turner, Department of Mathematics, Faulkner University, MontGOMERY AL, 36109, dturner@faulkner.edu 